\title{
Experimental investigation of two-bolt connections for high strength steel members
}

\author{
Yan-Bo Wanga, Yi-Fan Lyu a and Guo-Qiang $\mathrm{Li}^{\mathrm{b}}$ \\ ${ }^{a}$ College of Civil Engineering, Tongji University, China \\ ${ }^{\mathrm{b} S}$ State Key Laboratory for Disaster Reduction in Civil Engineering, Tongji University, China, 200092 \\ *corresponding author, e-mail address: ybwang@tongji.edu.cn
}

\begin{abstract}
This paper presents an experimental research on bearing-type bolted connections consisting of two bolts positioned perpendicular to the loading direction. A total of 24 connections in double shear fabricated from high strength steels with yield stresses of $677 \mathrm{MPa}$ and $825 \mathrm{MPa}$ are tested. Two failure modes as tearout failure and splitting failure are observed in experiments. The effect of end distance, edge distance, bolt spacing and steel grade on the failure mode and bearing behavior are discussed. For connection design with bolts positioned perpendicular to loading direction, it is further found that combination of edge distance and bolt spacing effectively determines the failure mode and ultimate load. The test results are compared with Eurocode3. An optimal combination of edge distance and bolt spacing as well as related design suggestion is thus recommended.
\end{abstract}

Keywords: High strength steel; two-bolt connection; tearout failure; splitting failure

\section{Introduction}

Bearing-type bolted connection is a widely used connection type in design of steel structures. The resistance of connection is provided by the bearing between bolt and plate. If bolt is designed sufficient to avoid shear failure, the resistance of connection is determined by the material strength of steel plate. High strength steel (HSS) with a nominal yield strength $\geqslant 460 \mathrm{MPa}$ [1] has improved material strength compared to normal strength steel. Thus, application of HSS into bearing-type bolted connection is a promising strategy, which will effectively increase the bearing resistance.

Experiment on single-bolt connection has been a popular method to test the bearing resistance of an individual bolt [2-4]. However, in multi-bolt connection, the applied load is carried by a group of bolts. Behavior of an individual bolt in multi-bolt connection will be affected by other bolts nearby, which is not considered in single-bolt connection. As a result, research on multi-bolt connection is necessary. The connection with two bolts positioned perpendicular to the loading direction is a basic form of multi-bolt connection. Study on this type of two-bolt connection can effectively test the bearing behavior of an individual bolt affected by another bolt positioned perpendicular to loading direction. Thus, the connection with two bolts positioned perpendicular to loading direction will be mainly discussed in this paper. This paper further provides an experimental analysis on 24 groups of connections with two bolts positioned perpendicular to loading direction. Two types of high strength steels with yield stress of $677 \mathrm{MPa}$ and $825 \mathrm{MPa}$ are used, which particularly supplements the data set for two-bolt connections made from high strength steels.

\section{Experimental work}

A total of 24 groups of two-bolt connections positioned perpendicular to loading direction are prepared for the research. Two high strength steels including Q550D and Q690D are used. The two HSS steels are produced by Wuyang Iron \& Steel Co., Ltd. Based on GB/T 228-2002 [5], standard tensile tests are conducted at room temperature. The measured material properties are listed in Table 1, where $f_{\mathrm{y}}$ is yield strength, $f_{\mathrm{u}}$ is tensile strength, $E$ is elasticity modulus, $\varepsilon_{\mathrm{u}}$ is the strain at tensile strength, $\Delta$ is the elongation 
ratio at fracture. Fig. 1 presents the measured stress-strain relationship for each high strength steel.

The two-bolt connection is named as TH connection, which is abbreviation for "Two-bolt, Horizontally positioned". Five main geometric parameters are displayed in Fig. 2, including end distance $e_{1}$, edge distance $e_{2}$, bolt spacing $p_{2}$, bolt hole diameter $d_{0}$ and plate thickness $t$. All the TH specimens are cited in terms of TH- $e_{1} / d_{0}-e_{2} / d_{0}-$ $p_{2} / d_{0}$-steel grade. The nominal and measured dimensions for each specimen are listed in Table 2. In Table 2, two different end distances $e_{1}$ as $1.2 d_{0}$ (minimum requirement in Eurocode 3 ) and $1.5 d_{0}$ are adopted. Six combinations of edge distance $e_{2}$ and bolt spacing $p_{2}$ are prepared for each $e_{1}$, as is listed in Table 2 .

Table 1 Measured material properties

\begin{tabular}{ccccccc}
\hline Steel Grade & $f_{y}(\mathrm{MPa})$ & $f_{u}(\mathrm{MPa})$ & $f_{y} / f_{u}$ & $E(\mathrm{GPa})$ & $\varepsilon_{u}$ & $\Delta(\%)$ \\
\hline Q550D & 677 & 757 & 0.894 & 205 & 0.0642 & 18.5 \\
Q690D & 825 & 859 & 0.960 & 203 & 0.0511 & 13.5 \\
\hline
\end{tabular}

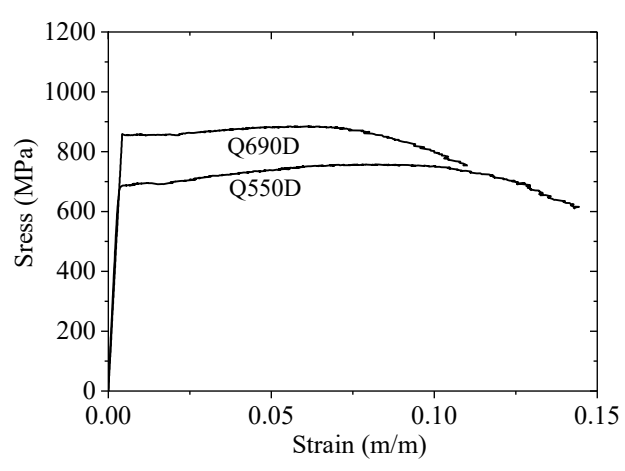

Fig. 1 Stress-strain relationship of steel plates

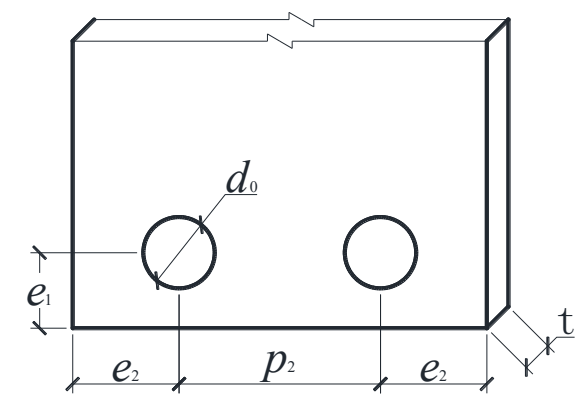

Fig. 2 Geometric parameters in TH connection

The tests are carried out on a hydraulic servocontrolled machine with loading capacity of $2000 \mathrm{kN}$. Experimental set-up is displayed in Fig. 3. High strength bolt M24 (bolt diameter $d=$ $24 \mathrm{~mm}$ ) with grade 12.9 is used and designed with adequate shear resistance to avoid bolt shear failure in experiments. No pretension of bolt is considered and a $1.6 \mathrm{~mm}$ gap is reserved, as is shown in Fig. 3. These two precautions ensures that load is primarily transferred by bearing. A $10 \mathrm{kN}$ load is applied and unloaded to make bolt shank bear on hole wall before formal loading. Then, the specimens are loaded at a prescribed rate of $1.5 \mathrm{~mm} / \mathrm{min}$ until final failure occurs. Two linearly variable displacement transducers (LVDTs) are positioned along both edges of the specimen to measure the bolt hole elongation and plate deformations in the force direction, as shown in Fig. 3.

Table 2 Geometrical dimensions (Unit: $\mathrm{mm}$ )

\begin{tabular}{cccccc}
\hline \multirow{2}{*}{ Specimen } & \multicolumn{5}{c}{ Nominal dimensions } \\
\cline { 2 - 6 } & $e_{1} / d_{0}$ & $e_{2} / d_{0}$ & $p_{2} / d_{0}$ & $d_{0}$ & $t$ \\
\hline TH-12-12-24 & 1.20 & 1.20 & 2.40 & 26 & 10 \\
TH-12-12-27 & 1.20 & 1.20 & 2.70 & 26 & 10 \\
TH-12-12-30 & 1.20 & 1.20 & 3.00 & 26 & 10 \\
TH-12-15-24 & 1.20 & 1.50 & 2.40 & 26 & 10 \\
TH-12-15-27 & 1.20 & 1.50 & 2.70 & 26 & 10 \\
TH-12-15-30 & 1.20 & 1.50 & 3.00 & 26 & 10 \\
TH-15-15-30 & 1.50 & 1.50 & 3.00 & 26 & 10 \\
TH-15-15-35 & 1.50 & 1.50 & 3.50 & 26 & 10 \\
TH-15-15-40 & 1.50 & 1.50 & 4.00 & 26 & 10 \\
TH-15-20-30 & 1.50 & 2.00 & 3.00 & 26 & 10 \\
TH-15-20-35 & 1.50 & 2.00 & 3.50 & 26 & 10 \\
TH-15-20-40 & 1.50 & 2.00 & 4.00 & 26 & 10 \\
\hline
\end{tabular}




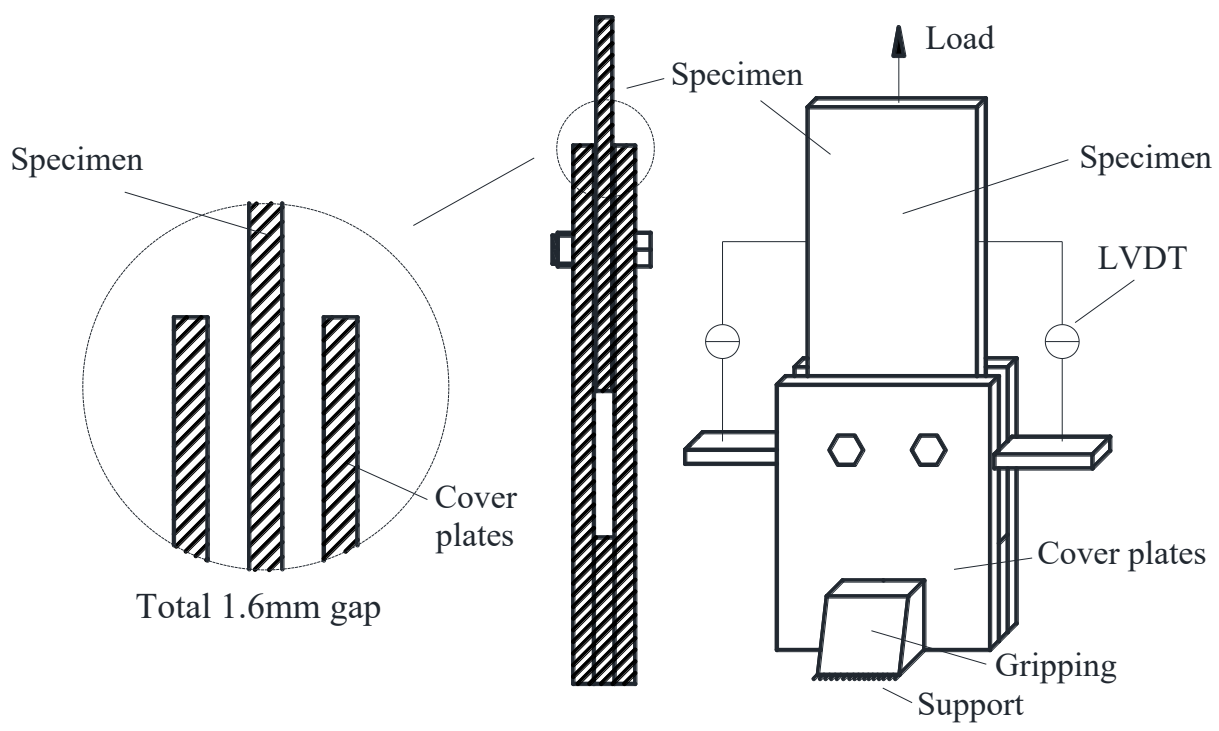

Fig. 3 Experimental set-up

\section{Test results and observations}

\subsection{Failure mode}

The comparison of failure modes of $\mathrm{TH}$ specimens for each high strength steel is shown in Fig. 4. Two profiles of failure modes associated with tearout and splitting failure are schematically shown in Fig. 5. The first three columns of each row in Fig. 4 show the experimental splitting failure and the second three columns display the tearout failure. In splitting failure, rotation of net cross-section exists, which is less significant in tearout failure. A comparison with the failue mode of single-bolt connection [2] is shown in Fig. 6. The single-bolt connections are made from the same steels in Table 1. With Fig. 4 to Fig. 6, the effect of the geometric parameters $\left(e_{1}, e_{2}\right.$ and $\left.p_{2}\right)$ and mechanical properties of steel plate are observed as follows.

(1) End distance $e_{1}$ : For specimens with $e_{1}=1.2 d_{0}$ or $1.5 d_{0}$, similar bolt hole elongation and shear fracture are observed, no matter what $e_{2}$ or $p_{2}$ are used. Compared to failure mode of single-bolt connection in Fig. 6, bolt hole elongation and profile of shear fracture display no significant difference.

(2) Edge distance $e_{2}\left(e_{1} / e_{2}\right)$ : For specimens with $e_{1}=1.2 d_{0}$ or $1.5 d_{0}$, two types of $e_{1} / e_{2}$ are used. For $e_{1} / e_{2}$ around 1.0 (e.g., TH-12-12-24-550: $e_{1}=1.2 d_{0}$ and $\left.e_{2}=1.2 d_{0}\right)$, significant rotation of net cross section is observed. For $e_{1} / e_{2}$ around 0.8 (e.g., TH-12-12-24-550: $e_{1}=1.2 d_{0}$ and $e_{2}=1.5 d_{0}$ ), no significant rotation of net cross section is found. The similar rotation of net cross-section can also be found in single-bolt connection with $e_{1}=1.5 d_{0}$ and $e_{2}=1.5 d_{0}$, as is shown in Fig. 6, indicating the similarity of bearing behavior between $\mathrm{TH}$ connection and the single-bolt connection.

(3) Bolt spacing $p_{2}$ : There are six values of $p_{2}$ increasing from $2.4 d_{0}$ to $4.0 d_{0}$ for each high strength steel. The value of $2.4 d_{0}$ is the minimum value accepted in Eurocode3. Result in Fig. 4 indicates that effect of $p_{2}$ on bolt hole elongation or shear fracture is negligible when tearout or splitting failure occurs.

(4) Steel grade: The TH specimens with identical dimensions but different steel grades show similar failure modes.

\subsection{Load-displacement curve and ultimate load}

The load-displacement curves of $\mathrm{TH}$ specimens are displayed in Fig. 7. Two clusters of curves are observed. It can be found that specimens with $e_{1}=1.5 d_{0}$ have obviously increased ultimate load as well as deformation capacity compared to specimens with $e_{1}=1.2 d_{0}$. For specimens with the same $e_{1}$, smaller edge distance $e_{2}$ will cause slight reduction of ultimate strength but no significant reduction of deformation capacity, as is shown in Fig. 7. Effect of steel grade on the profile of loaddisplacement curve for $\mathrm{TH}$ specimens is negligible. Smooth descending stage is observed in each load-displacement curve, no matter what steel grade is used. Smooth descending stage is 
observed in each load-displacement curve, no matter which steel grade is used.

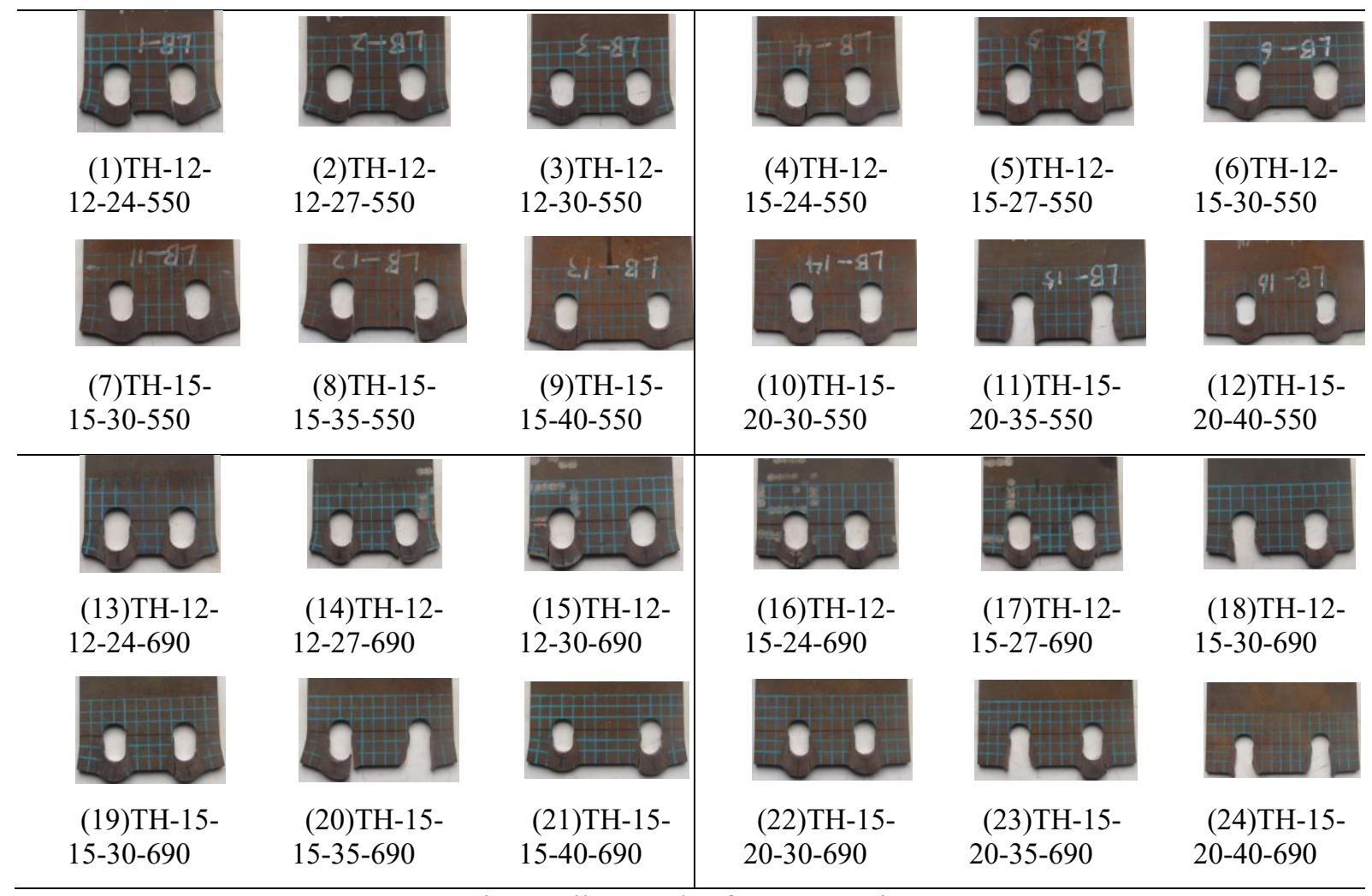

Fig. 4 Failure mode of TH connection

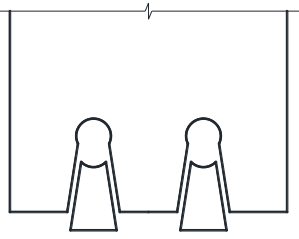

Tearout failure

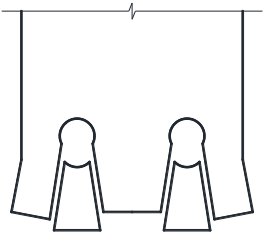

Splitting failure
Fig. 5 Typical failure modes

The measured ultimate load $F_{\mathrm{u}}$, corresponding deformation $D_{\mathrm{u}}$ and the normalized ultimate load $\left(F_{\mathrm{u}} / f_{\mathrm{u}} d t, d\right.$ is the diameter of bolt shank) for each $\mathrm{TH}$ specimen are summarized in Table 3. For specimens with the same end distance $e_{1}$, similar values of $D_{\mathrm{u}}$ are observed no matter what combination of $e_{2}$ and $p_{2}$ is used. For different high strength steels, similarity of the normalized ultimate load is found for each TH specimen. Comparison with result of single-bolt connection is also conducted in Table 3. The ultimate load of single-bolt connection $\left(F_{\text {uSingle }}\right)$ in Table 3 is experimental

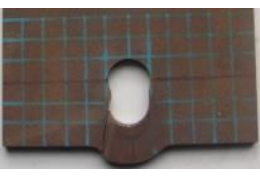

(a) $e_{1}=1.5 d_{0}$, $e_{2}=3.0 d_{0}, \mathrm{Q} 550 \mathrm{D}$

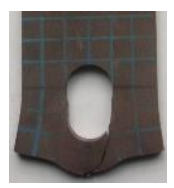

(d) $e_{1}=1.5 d_{0}$, $e_{2}=1.5 d_{0}, \mathrm{Q} 550 \mathrm{D}$

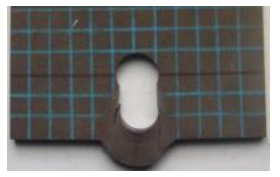

(b) $e_{1}=1.5 d_{0}$, $e_{2}=3.0 d_{0}, \mathrm{Q} 690 \mathrm{D}$

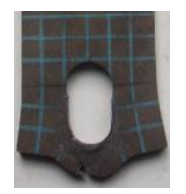

(e) $e_{1}=1.5 d_{0}$, $e_{2}=1.5 d_{0}, \mathrm{Q} 690 \mathrm{D}$
Fig. 6 Failure mode of single-bolt connection

result from [2], which is also conducted by the authors of this paper. It can be found that both $F_{\mathrm{u}}$ of TH connection is close to sum of two singlebolt connections with the same $e_{1}$, while $D_{\mathrm{u}}$ shows similarity between the two types of connections. The observation in Table 3 indicates that an individual bolt in $\mathrm{TH}$ connection has the similar bearing behavior compared to single-bolt connection with the same $e_{1}$ 

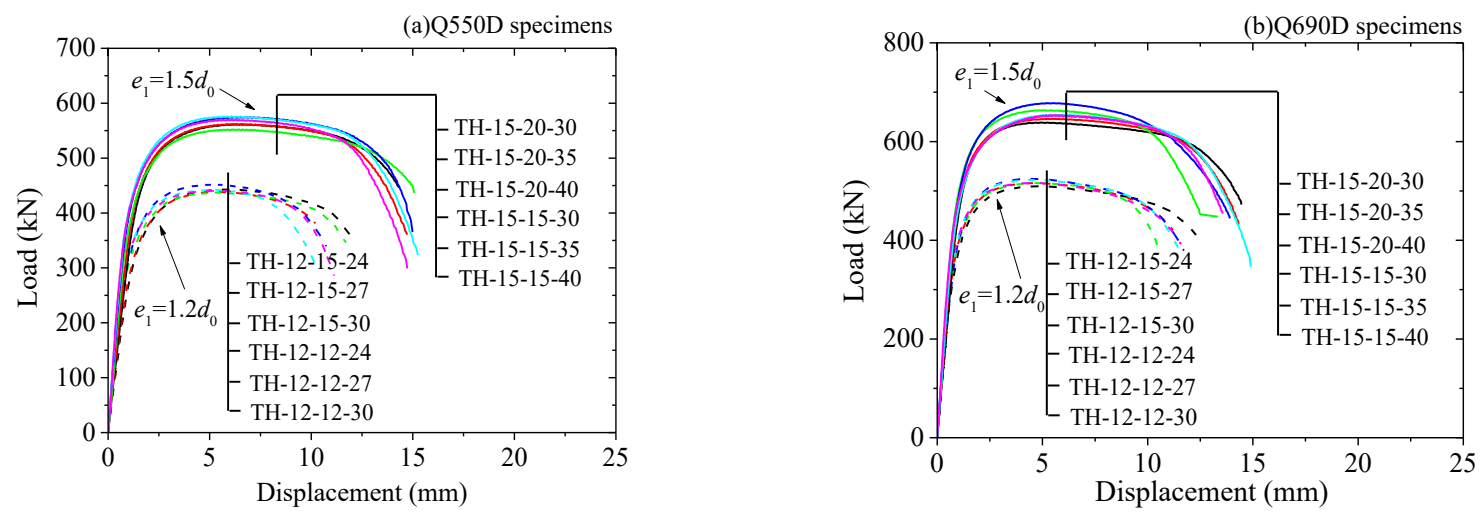

Fig. 7 Load-displacement curves

Table 3 Ultimate load of TH connection and comparison with single-bolt

\begin{tabular}{cccccc}
\hline Specimen & $F_{\mathrm{u}}(\mathrm{kN})$ & $F_{\mathrm{u}} / f_{\mathrm{u}} d t$ & $D_{\mathrm{u}}(\mathrm{mm})$ & $F_{\mathrm{u}} /\left(2 * F_{\mathrm{uSingle}}\right)$ & $D_{\mathrm{u}} /\left(D_{\mathrm{uSingle}}\right)$ \\
\hline TH-12-12-24-550 & 443 & 2.44 & 5.25 & 0.98 & 1.04 \\
TH-12-12-27-550 & 439 & 2.41 & 5.26 & 0.97 & 1.04 \\
TH-12-12-30-550 & 438 & 2.41 & 5.37 & 0.97 & 1.07 \\
TH-12-15-24-550 & 452 & 2.49 & 5.31 & 1.00 & 1.05 \\
TH-12-15-27-550 & 442 & 2.43 & 5.29 & 0.98 & 1.05 \\
TH-12-15-30-550 & 441 & 2.43 & 5.11 & 0.97 & 1.01 \\
TH-15-15-30-550 & 561 & 3.09 & 6.66 & 0.96 & 0.96 \\
TH-15-15-35-550 & 562 & 3.09 & 6.64 & 0.96 & 0.96 \\
TH-15-15-40-550 & 552 & 3.04 & 6.38 & 0.95 & 0.92 \\
TH-15-20-30-550 & 575 & 3.16 & 6.63 & 0.98 & 0.95 \\
TH-15-20-35-550 & 576 & 3.17 & 6.44 & 0.99 & 0.93 \\
TH-15-20-40-550 & 570 & 3.14 & 6.34 & 0.98 & 0.91 \\
\hline TH-12-12-24-690 & 511 & 2.43 & 4.55 & 1.03 & 1.01 \\
TH-12-12-27-690 & 516 & 2.45 & 4.57 & 1.04 & 1.01 \\
TH-12-12-30-690 & 516 & 2.45 & 4.64 & 1.04 & 1.03 \\
TH-12-15-24-690 & 525 & 2.49 & 4.60 & 1.05 & 1.02 \\
TH-12-15-27-690 & 523 & 2.49 & 4.46 & 1.05 & 0.99 \\
TH-12-15-30-690 & 516 & 2.45 & 4.61 & 1.04 & 1.02 \\
TH-15-15-30-690 & 639 & 3.04 & 5.50 & 0.96 & 1.02 \\
TH-15-15-35-690 & 646 & 3.07 & 5.52 & 0.97 & 1.03 \\
TH-15-15-40-690 & 663 & 3.15 & 5.63 & 1.00 & 1.05 \\
TH-15-20-30-690 & 678 & 3.22 & 5.41 & 1.02 & 1.01 \\
TH-15-20-35-690 & 654 & 3.11 & 5.38 & 0.99 & 1.00 \\
TH-15-20-40-690 & 654 & 3.11 & 5.55 & 0.98 & 1.03 \\
\hline
\end{tabular}

\section{Comparison with design code}

According to current Eurocode3 [6], the bearing resistance for an individual fastener is designated as Eq. (1-5). For group of fasteners, if bolt shear failure does not happen, bearing resistance of the whole connection is sum of each bolt.

$$
F_{b, R d}=\alpha_{b} k_{1} f_{u} d t \leq 2.5 f_{u} d t
$$

$$
\begin{aligned}
& \alpha_{b}=\min \left(\frac{e_{1}}{3 d_{0}} ; \frac{f_{u b}}{f_{u}} ; 1\right) \\
& k_{1}=\min \left(2.8 \frac{e_{2}}{d_{0}}-1.7 ; 2.5\right)
\end{aligned}
$$

For edge bolts 


$$
\begin{aligned}
& \alpha_{b}=\min \left(\frac{p_{1}}{3 d_{0}}-\frac{1}{4} ; \frac{f_{u b}}{f_{u}} ; 1\right) \\
& k_{1}=\min \left(1.4 \frac{p_{2}}{d_{0}}-1.7 ; 2.5\right)
\end{aligned}
$$

Where, $f_{u b}$ is the ultimate tensile strength of bolt

The formula of bearing resistance in current Eurocode 3 can be divided into two parts [6]. The first part is $\alpha_{b} k_{1} f_{u} d t$, which considers the condition of tearout failure. The second part is $2.5 f_{u} d t$, which considers the limit on bolt hole elongation. Prediction of Eurocode 3 for specimens in tearout failure from this paper is displayed in Fig. 8. Two clusters of data are observed in Fig. 8. The first cluster of data has the relative difference around $20 \%$ and the second around $40 \%$. Feature of the first data cluster is $e_{2} \geqslant 1.5 d_{0}$ and $p_{2} \geqslant 3.0 d_{0}$, while feature of the second data cluster is the opposite. The $45 \%$ relative difference is due to the parameter $k_{1}$ in Eq. (3). If $e_{2}<1.5 d_{0}$ or $p_{2}<3.0 d_{0}$, the parameter $k_{1}$ is smaller than 2.5, which incorporates a reduction into the bearing resistance. However, it can be found in Fig. 8 that prediction of Eurocode 3 is already conservative without the reduction. As a result, the parameter $k_{1}$ is recommended to be 2.5 for all the values of edge distance $e_{2}$. Furthermore, the bearing resistance in Eurocode 3 aims for tearout failure and limit on bolt hole elongation. Thus, a geometry check or the optimal geometry as $2 e_{2} / p_{2}=1$ is suggested in order to exclude the possible mixed failure.

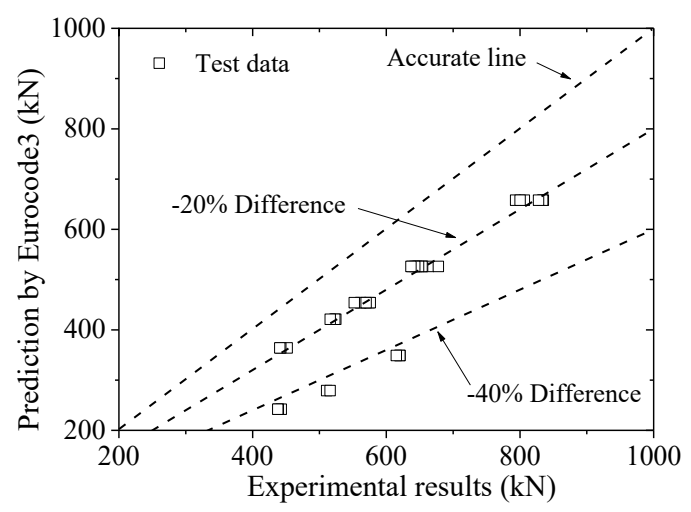

Fig. 8 Prediction of Eurocode 3 for TH connection

\section{Conclusions}

An experimental and numerical study on twobolt connections perpendicular to loading direction made from two grades of high strength steel (Q550D and Q690D) are conducted. Two failure modes as tearout failure and splitting failure are observed in the experiments. Parameter end distance $e_{1}$ has significant effect on the ultimate load and deformation capacity. Significant rotation of net cross-section and slight reduction of ultimate load are observed in specimens with $e_{1} / e_{2}$ around 1.0. In addition, bolt spacing $p_{2}$ and steel grade has negligible effect on the failure mode. Comparison with current Eurocode 3 is conducted by this paper. The reduction of bearing resistance considered in the parameter $k_{1}$ is found unnecessary, since the designed resistance is already conservative without the reduction. A geometry check is recommended in order to exclude the possible mixed failure.

\section{Acknowledgement}

The authors would like to acknowledge the funding support by National Natural Science Foundation of China, Grant No. 51408428.

\section{References}

[1] Shi G, Hu F, Shi Y. Recent research advances of high strength steel structures and codification of design specification in China. INT J STEEL STRUCT 2014;14:873-887.

[2] Wang Y-B, Lyu Y-F, Li G-Q. Behavior of single bolt bearing on high strength steel plate. Journal of Constructional Steel Research 2017;137:1930.

[3] Može P, Beg D. High strength steel tension splices with one or two bolts. Journal of Constructional Steel Research 2010;66:1000-1010.

[4] Aalberg A, Larsen PK. The effect of steel strength and ductility on bearing failure of bolted connections. The third European conference on steel structures: proceedings of the 3rd European conference on steel structures. Universidad de Coimbra 2002.p.869-878

[5] National Standardization Technical Committees, GB/T 228-2002 Metallic materials: tensile testing at ambient temperature, China Standard Press, Beijing, 2002 (in Chinese)

[6] CEN. Eurocode 3: Design of steel structures, Part 1-8: Design of joints, EN 1993-1-8. Brussels; European Committee for Standardization. 2005. 